\title{
Un diagnóstico no pensado: toxicidad serotoninérgica secundaria a interacción medicamentosa. Caso clínico
}

\author{
An unintended diagnosis: Serotonergic toxicity secondary to drug interactions. \\ Case reports
}

\author{
Dra. Elda G. Cargnel ${ }^{a}$, Dra. Patricia C. Cardoso ${ }^{a}$ Dr. Julián Irigoyen ${ }^{a}$ y Dra. Sol García Puglisia.
}

\section{RESUMEN}

La toxicidad serotoninérgica es un trastorno con potencial riesgo de vida asociado con un incremento de la actividad serotoninérgica en el sistema nervioso central. Se observa con el uso terapéutico o sobredosis intencional de medicamentos e interacciones inadvertidas (inhibidores selectivos de la recaptación de serotonina-isoniacida). Aunque esta patología está incrementándose, todavía no es bien reconocida por los médicos y sus manifestaciones pueden ser erróneamente atribuidas a otras causas. El objetivo de este artículo es presentar un caso clínico, colaborar con el diagnóstico y mejorar el cuidado de estos pacientes.

Palabras clave: sindrome de la serotonina, agentes de serotonina, isoniacida, interacciones medicamentosas.

\begin{abstract}
Serotonin toxicity is a potentially life-threatening condition associated with increased serotonergic activity in the central nervous system. It is seen with therapeutic medication use, intentional self-poisoning and inadvertent interactions (SSRIisoniazid). Although this pathology is increasingly common, it is not well recognized by physicians and manifestations may be wrongly attributed to another cause. The aim of this paper is to describe the clinical picture of a patient, to collaborate on diagnosis and to improve medical care of these patients. Key words: serotonin syndrome, serotonergic agents, isoniazid, drug interactions.
\end{abstract}

http:/ / dx.doi.org/10.5546/ aap.2018.e115

Cómo citar: Cargnel EG, Cardoso PC, Irigoyen J, et al. Un diagnóstico no pensado: toxicidad serotoninérgica secundaria a interacción medicamentosa. Caso clínico. Arch Argent Pediatr 2018;116(1):e115-e120.

a. Unidad de Toxicología, Hospital de Niños

"Ricardo Gutiérrez", Ciudad Autónoma de Buenos Aires.

Correspondencia:

Dra. Elda G. Cargnel: ecargnel@gmail.com

Financiamiento: Ninguno.

Conflicto de intereses: Ninguno que declarar.

Recibido: 5-5-2017

Aceptado: 17-8-2017

\section{INTRODUCCIÓN}

El espectro de toxicidad serotoninérgica (TS) es un cuadro potencialmente mortal, consecuencia del exceso de serotonina en el sistema nervioso central (SNC) y periférico. ${ }^{1} \mathrm{Se}$ lo relaciona con el uso de inhibidores selectivos de la recaptación de serotonina (ISRS) y puede desencadenarse luego de una única dosis terapéutica o por una interacción medicamentosa. Otras drogas pueden ocasionarlo: inhibidores de la monoaminooxidasa, antidepresivos tricíclicos, opioides, antirretrovirales, antieméticos, anfetaminas, metilendioximetanfetamina (éxtasis) y suplementos dietarios. ${ }^{1,2}$

En 1960, Oates y Sjostrand, observan el exceso de serotonina en el sistema nervioso central como un problema clínico. En 1991, Sternbach ${ }^{3}$ revisa 38 casos reportados y elabora una serie de criterios diagnósticos que define como síndrome serotoninérgico. Otras notificaciones basadas en los criterios de Sternbach incluyen otros síntomas y signos. ${ }^{4}$

Es mejor pensar el exceso de serotonina como un espectro de toxicidad con una clara importancia pronóstica que como una entidad clínica definida. De ahora en adelante, se hará referencia a los efectos tóxicos del exceso de serotonina como TS. ${ }^{5}$

\section{CASO CLÍNICO}

Desde la Unidad de Toxicología, se desea ejemplificar la dificultad diagnóstica que se observa. Es una paciente de 17 años, con depresión mayor y trastorno de conducta alimentaria, tratada con $100 \mathrm{mg} /$ día de sertralina, $75 \mathrm{mg} /$ día de quetiapina y $1 \mathrm{mg} /$ día de clonazepam. Es internada por una ingesta masiva voluntaria de clonazepam.

El Servicio de Salud Mental aumenta las dosis de sertralina y quetiapina hasta $150 \mathrm{mg}$ / día y $200 \mathrm{mg} /$ día, respectivamente.

Tiene contacto con un paciente tuberculoso; comienza el día 40 de internación la profilaxis con $300 \mathrm{mg} /$ día de isoniacida. Al mismo tiempo, se 
desciende progresivamente el clonazepam hasta suspender (véase la Figura1).

Cuarenta y ocho horas después de esta suspensión, asintomática y con buena evolución, se le otorga permiso de salida durante la tarde. $\mathrm{Al}$ regreso, refiere debilidad y se observa palidez generalizada, diaforesis e hiporreactividad con disminución global de la fuerza muscular, midriasis bilateral arreactiva, con parpadeo unilateral izquierdo. Presenta ventilación no efectiva, saturación arterial de oxígeno de $65 \%$ al respirar aire ambiental, con una buena entrada de aire bilateral, frecuencia cardíaca (FC) de 80 latidos/min, tensión arterial (TA) de $130 / 80 \mathrm{mmHg}$, temperatura de $36,6{ }^{\circ} \mathrm{C}$, glucemia de $120 \mathrm{mg} / \mathrm{dl}$. No responde a estímulos dolorosos; se inician medidas de reanimación. Presenta mejoría clínica, con saturación de 98\%, hemodinámicamente compensada. Por un análisis de laboratorio, evidencia acidosis respiratoria.

A continuación, presenta un episodio de hiperextensión del cuello, fijación de la mirada, movimientos de chupeteo y flexoextensión de los miembros, que se interpreta como una convulsión tónico-clónica generalizada, que cede con lorazepam endovenoso. Se resuelve con restitución ad integrum y amnesia total del episodio.

La evaluación cardiológica, tomografía axial computada (TAC) de cerebro y electroencefalograma de sueño están dentro de los parámetros normales. El equipo médico interpreta el cuadro clínico como efecto adverso por isoniacida y la suspende.

Un análisis retrospectivo los hace desestimar este efecto adverso, que se resuelve con benzodiacepinas, sin el aporte de vitamina B6, y se reimplementa el tratamiento.

Tras 48 horas de reiniciado, presenta nuevamente movimientos tónico-clónicos generalizados, diaforesis, palidez y cianosis peribucal. Se observa parpadeo bilateral, pupilas midriáticas hiporreactivas y retrodesviación de la mirada. El episodio no cede con benzodiacepinas; se considera estatus convulsivo y se impregna con $20 \mathrm{mg} / \mathrm{kg}$ de difenilhidantoína, con buena respuesta. Presenta FC de 140 latidos/min, frecuencia respiratoria (FR) de $12 \mathrm{resp} . / \mathrm{min}$, TA de $130 / 65 \mathrm{mmHg}$, temperatura de $37,5^{\circ} \mathrm{C}$, saturación de oxígeno de $98 \%$, glucemia de 103 $\mathrm{mg} / \mathrm{dl}$. Durante $72 \mathrm{~h}$, persiste con somnolencia, amnesia y trastorno de la memoria reciente, hiperreflexia, bradipsiquia, leve dismetría, temblor fino distal, pupilas midriáticas reactivas e inyección conjuntival.

Se decide la suspensión definitiva de la isoniacida y continuar con quetiapina y sertralina (véase la Figura 1).

Ante la duda diagnóstica, se consulta a la Unidad de Toxicología, donde se revisa el caso y

FIGURA 1. Tratamiento recibido por la pacientedurante su internación. Las líneas verticales gruesas marcan los episodios convulsivos

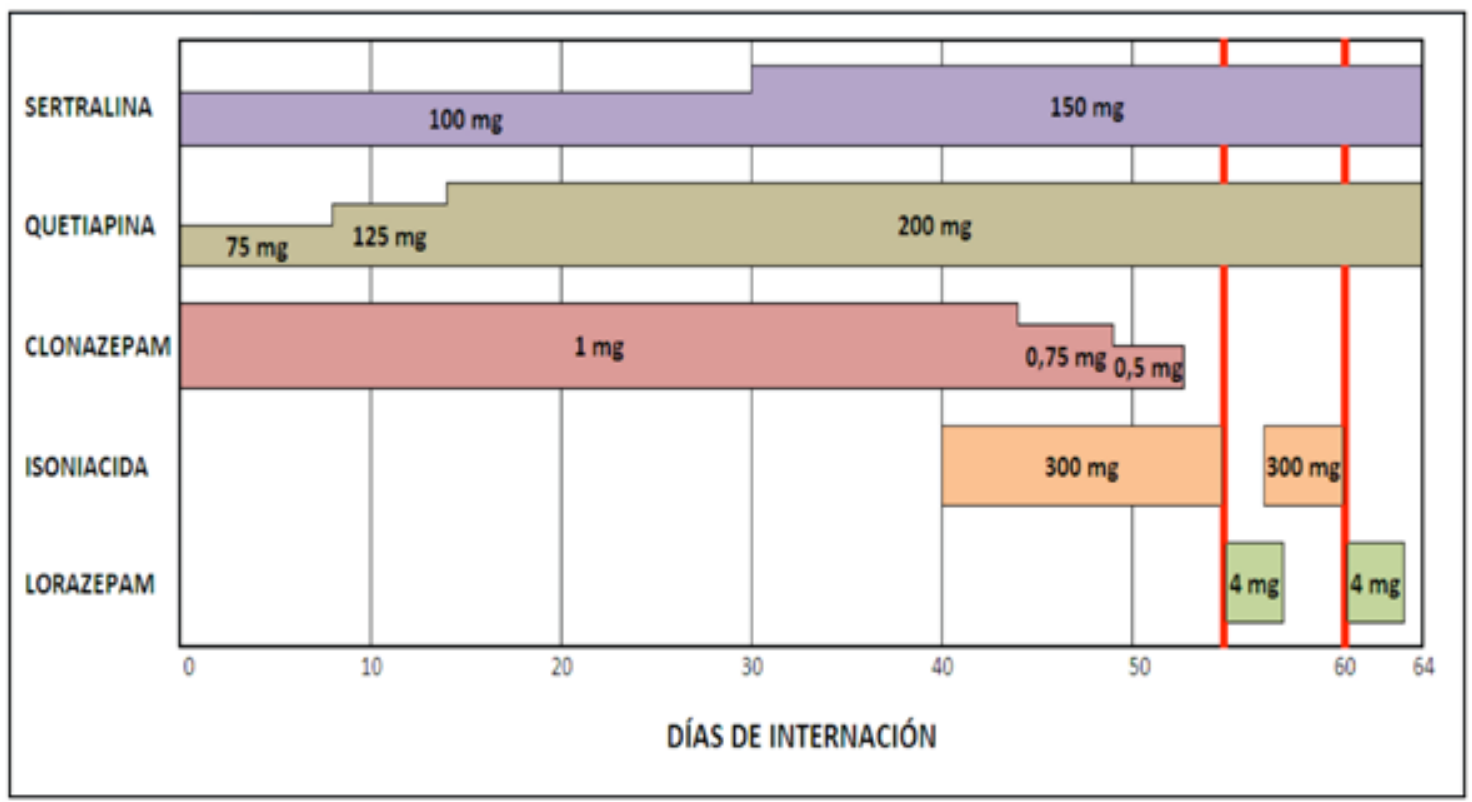


se concluye que el cuadro se debe a TS secundaria a interacción medicamentosa.

Luego de un año, no ha vuelto a presentar síntomas compatibles con TS y continúa con la misma medicación psicoactiva y bajo seguimiento de Salud Mental.

\section{DISCUSIÓN}

Desde la Unidad de Toxicología, se estudió si las manifestaciones eran por causa toxicológica o patología orgánica. Se descartó, por electroencefalograma de sueño normal, un foco epileptógeno y, por anamnesis dirigida y pesquisa de drogas en orina, drogas de abuso y otros tóxicos.

Se evaluó la posibilidad de que las manifestaciones fueran una reacción adversa medicamentosa (quetiapina, sertralina, isoniacida y retiro del clonazepam) o sobredosis de isoniacida. ${ }^{6}$ Esto no explicaría la persistencia del temblor, midriasis e hiperreflexia $72 \mathrm{~h}$ después de la convulsión, pero sí pueden corresponderse con un nivel elevado de serotonina en el SNC. ${ }^{7}$

Se consideró el síndrome neuroléptico maligno (SNM) como diagnóstico diferencial.

Para establecer causalidad, se tomaron en cuenta la relación entre la cronología de las manifestaciones clínicas, las modificaciones en el esquema de medicación y se utilizaron los criterios de imputabilidad de Naranjo. ${ }^{8}$ Esta relación orientó a deducir que se estaba en presencia de una interacción medicamentosa, que se volvió a manifestar clínicamente al reexponerla a la isoniacida.

Recibía habitualmente sertralina, que aumentaba la concentración de serotonina en la brecha sináptica. Este estímulo se vio potenciado al introducir isoniacida, por su efecto inhibidor de la monoaminooxidasa (IMAO) débil, que resultaba en una menor degradación de la serotonina. ${ }^{9}$ Los síntomas no se manifestaron hasta la suspensión del clonazepam, que contrarrestaba la aparición de TS, que comprendía una tríada que incluía alteraciones neuromusculares, hiperactividad autonómica y alteraciones en el estatus mental.
La detección temprana es importante, ya que la mayoría de los casos se resuelve con la suspensión de la medicación implicada. ${ }^{1,2,7}$

Si bien la TS puede deberse a monoterapia con un agonista serotoninérgico (en dosis terapéuticas o sobredosis), los casos más graves suelen ocurrir por una interacción medicamentosa. ${ }^{10}$

Pese al conocimiento de esta toxicidad, al médico clínico le resulta dificultoso llegar al diagnóstico, pues los signos y síntomas son inespecíficos, proteiformes y presentan diferentes grados de gravedad. ${ }^{11}$

Para facilitar su detección, se elaboraron diferentes escalas con criterios diagnósticos.

En 1991, Sternbach revisó 38 casos reportados y estableció criterios basados en las manifestaciones clínicas más frecuentemente observadas, con una sensibilidad del $75 \%$ y especificidad del $96 \%$.

Hegerl y col. modificaron los criterios diagnósticos propuestos por Sternbach y desarrollaron una escala que evaluaba 9 ítems. ${ }^{12}$

En el año 2003, Dunkley y col., estudiaron a 2222 pacientes admitidos al Servicio de Toxicología (Hunter Area Toxicology Service) y desarrollaron un método diagnóstico con mayor sensibilidad y especificidad ( $84 \%$ y $97 \%$, respectivamente). Debido a la cantidad de pacientes evaluados y teniendo en cuenta que la escala fue validada en el contexto de un servicio especializado en toxicología, se considera que los criterios de Hunter son actualmente los más adecuados para diagnosticar TS. ${ }^{13}$

Si se comparan las escalas, considerando los criterios de Sternbach (Tabla 1), la paciente cumplía con más de tres de las características clínicas mínimas requeridas: alteración del estatus mental, hiperreflexia, diaforesis, temblor y mioclonía ocular (parpadeo involuntario). En la de Hegerl, que considera diagnóstico de TS un puntaje de 6 o más, nuestra paciente sumaba un puntaje de 9 (Tabla 2).

Para las reglas de decisión de Hunter, el clonus ocular con diaforesis o temblor e hiperreflexia, en un paciente que recibe un agonista serotoninérgico, es diagnóstico de TS (Figura 2). El clonus (espontáneo, inducible y

TABLA 1. Criterios de Sternbach para definir un sindrome serotoninérgico (1991)

1. En un paciente bajo tratamiento con un agente serotoninérgico, al menos, tres de las siguientes características clínicas deben estar presentes: (a) alteraciones del estatus mental (confusión, hipomanía); (b) agitación; (c) mioclonías; (d) hiperreflexia; (e) diaforesis; (f) escalofríos; (g) temblores; (h) diarrea; (i) incoordinación; (j) fiebre.

2. Se deben descartar otras etiologías que expliquen el cuadro.

3. Ausencia de tratamiento con neurolépticos previo al inicio de los síntomas. 
ocular) es el signo más relevante en el estudio que desarrolló los criterios de Hunter.

La fiebre elevada $\left(>38^{\circ} \mathrm{C}\right)$ y la hipertonicidad/ rigidez se añaden en el último paso del árbol decisorio. Si bien no son tan precisos, su presencia caracteriza los cuadros graves y con riesgo de vida. Demostrar clonus e hiperreflexia es dificultoso con rigidez grave. La presencia de fiebre elevada e hipertonía/rigidez implican un alto riesgo de progresión del compromiso respiratorio y requieren de una intervención urgente.
Se observó midriasis en el 31,6\% de los pacientes y taquicardia en el $40 \%$. Aunque esta última es un hallazgo común en otras intoxicaciones, puede ser de utilidad para determinar, en este caso, la mejoría y la respuesta al tratamiento.

En adolescentes que reciben una combinación de fármacos IRS más un neuroléptico, se debe considerar el diagnóstico diferencial con SNM, ya que difieren en su morbimortalidad y tratamiento.

Los criterios para el SNM, de acuerdo con el Diagnostic and Statistical Manual of Mental Disorders

TABLA 2. Criterios de Hegerl (escala del sindrome serotoninérgico)

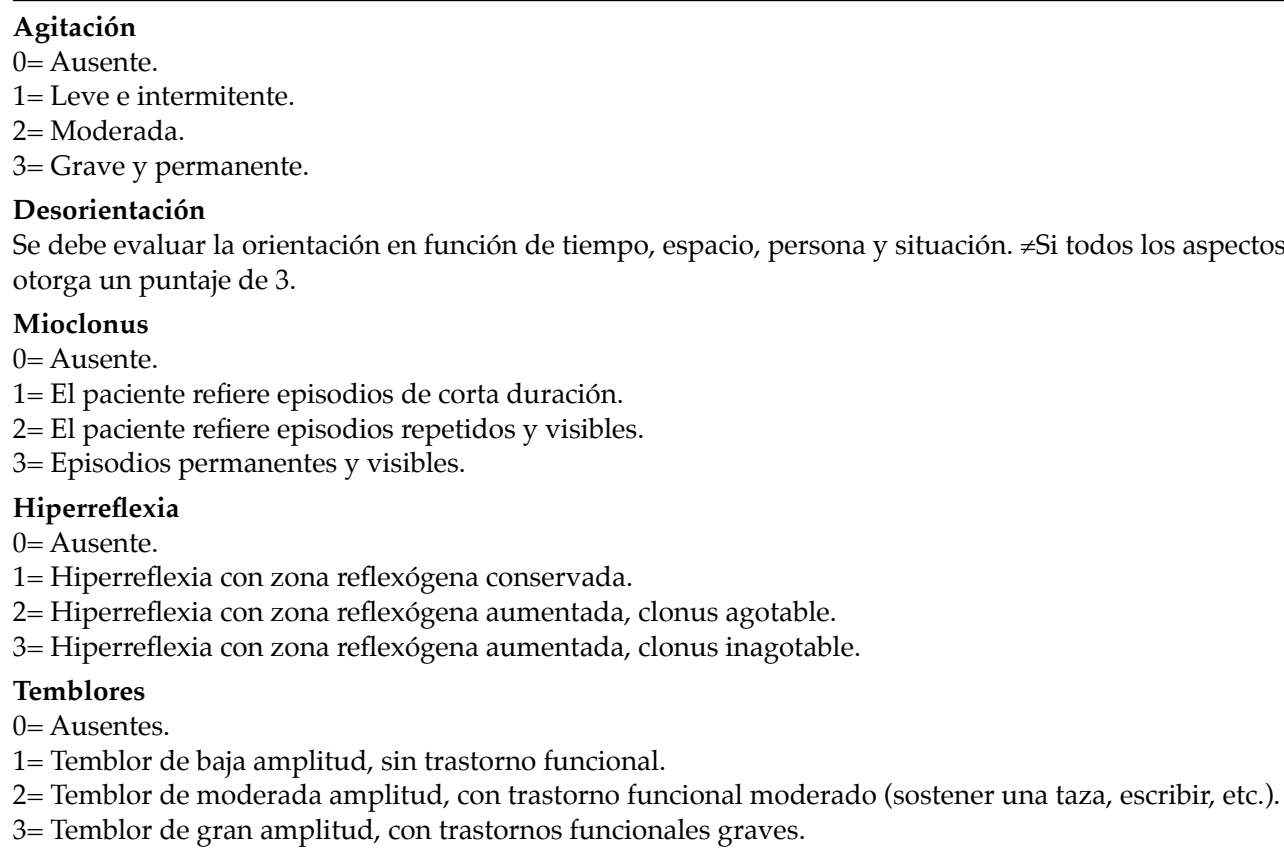

\section{Mareos}

$0=$ Ausente.

$1=$ Leve $\mathrm{e}$ intermitente.

$2=$ Persistentes, $\sin$ trastorno funcional.

$3=$ Persistente, con afectación funcional.

\section{Hipertermia (sublingual)}

$0=<37^{\circ} \mathrm{C}$

$1=37-37,9^{\circ} \mathrm{C}$.

$2=38-38,9^{\circ} \mathrm{C}$.

$3=>39^{\circ} \mathrm{C}$.

\section{Sudoración (en reposo, a temperatura ambiente)}

$0=$ Ausente.

$1=$ Sensación subjetiva de aumento de la sudoración.

$2=$ Piel húmeda.

$3=$ Sudoración profusa.

\section{Diarrea}

$0=$ Ausente.

$1=$ Consistencia de las heces disminuida, pero frecuencia conservada.

$2=$ Heces líquidas y / o una frecuencia de 1-3/día.

$3=$ Heces líquidas con frecuencia $>3 /$ día. 
5 (DSM-5), son hipertermia, rigidez, creatinafosfoquinasa $(\mathrm{CPK})>4$ veces el límite superior, cambios en el estatus mental (delirio, alteración de la conciencia) y activación autonómica (taquicardia, taquipnea, diaforesis, hipertensión arterial, incontinencia urinaria, palidez).

La fiebre es usualmente elevada, sin fluctuaciones importantes y con pobre respuesta a los antipiréticos convencionales. La rigidez muscular es generalizada, simétrica y va desde leve aumento del tono muscular a rigidez extrema con opistótonos. Pueden existir incrementos focales en el tono muscular con blefaroespasmo, crisis oculogiras, nistagmus, trismus, disartria, afonía o disfagia. El delirio es un síntoma distintivo, con fluctuaciones en el grado de conciencia, desorientación y agitación psicomotriz. La disautonomía se manifiesta como inestabilidad en la frecuencia cardíaca, hipertensión lábil y diaforesis extrema, que suele presentarse como "untuosa". En el análisis de laboratorio, la CPK es habitualmente mayor de $600 \mathrm{UI} / \mathrm{L}$ y se observa leucocitosis. ${ }^{14}$

El tratamiento de la TS consiste en discontinuar el estímulo serotoninérgico, suspender la/s droga/s que lo provoquen. Para revertir los síntomas, se deben utilizar benzodiacepinas.

Otras medidas (hidratación, correcciones del medio interno, anticonvulsivantes, asistencia respiratoria mecánica, etc.) se indican según la evolución clínica.

En casos graves, se utiliza ciproheptadina, un antagonista 5-HT2A (receptor serotoninérgico que estaría implicado en la fisiopatología de la TS), si bien no hay evidencia científica con respecto a sus beneficios.

FIGURA 2. Reglas de decisión de Hunter en presencia de un agente serotoninérgico

Reglas de decisión de Hunter para síndrome serotoninérgico

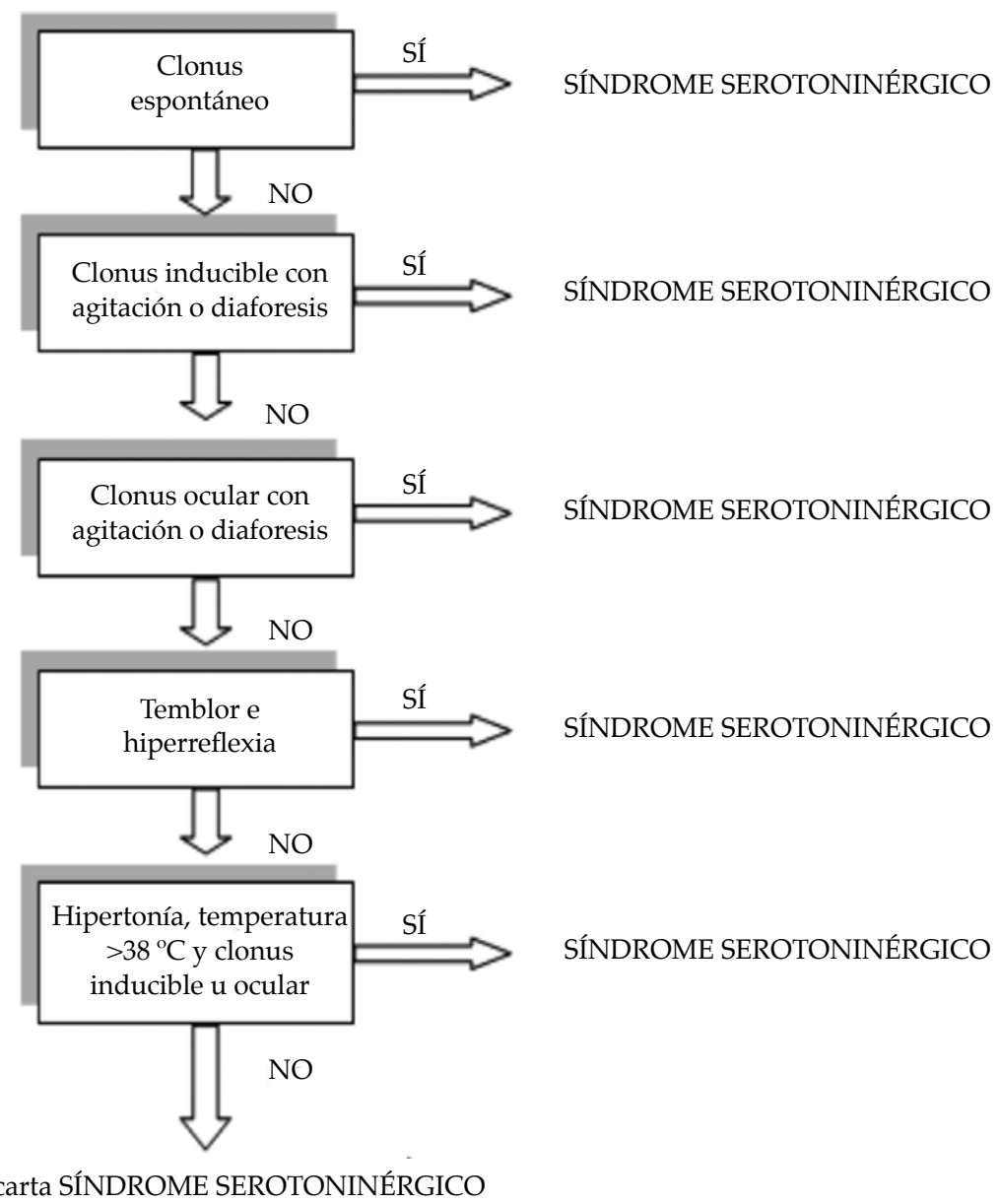


La TS no es un evento idiopático, sino un efecto adverso evitable, de allí la importancia de su detección temprana. Desafortunadamente, no existen estudios complementarios específicos para un diagnóstico de certeza; por lo tanto, el diagnóstico es clínico y por exclusión. ${ }^{15}$

\section{REFERENCIAS}

1. Ables AZ, Naqubili R. Prevention, recogniton, and management of serotonin syndrome. Am Fam Physician 2010;81(9):1139-42.

2. Boyer EW, Shannon M. The Serotonin Syndrome. N Engl J Med 2005;352(11):1112-20.

3. Sterbach H. The Serotonin Syndrome. Am J Pshychiatry. 1991;148(6):705-13.

4. Mackay FJ, Dunn NR, Mann RD. Antidepressants and the serotonin syndrome in general practice. Br J Gen Pract 1999;49(448):871-4.

5. Dvir Y, Smallwood, P. Serotonin syndrome: a complex but easily avoidable condition. Gen Hosp Psychiatry 2008;30(3):284-7.

6. Ebadi M, Gessert, GF, Al-Sayegh A. Drug-Pyridoxal Phosphate Interactions. Q Rev Drug Metab Drug Interact 1982;4(4):289-331.

7. Radomski JW, Dursun SM, Reveley MA, et al. An exploratory approach to the serotonin syndrome: an update of clinical phenomenology and revised diagnostic criteria. Med Hypotheses 2000;55(3):218-24.

8. Naranjo CA, Busto U, Sellers EM, et al. A method for estimating the probability of adverse drug reactions. Clin Pharmacol Ther 1981;30(2):239-45.

9. Polasek TM, Elliot DJ, Somogyi AA, et al. An evaluation of potential mechanism-based inactivation of human drug metabolizing cytochromes $\mathrm{P} 450$ by monoamine oxidase inhibitors, including isoniazid. Br J Clin Pharmacol 2006;61(5):570-84.

10. Young P, Finn BC, ÁlvarezF, etal.Síndromeserotoninérgico. Presentación de cuatro casos y revisión de la literatura. $A n$ Med Intern (Madrid) 2008;25(3):125-30.

11. Gillman, P. Review of Serotonin Toxicity Data:Implications for the Mechanisms of Antidepressant Drug Action. Biol Psychiatry 2006;59(11):1046-51.

12. Hegerl U, Bottlender R, Gallinat J, et al. The serotonin syndrome scale: first results on validity. Eur Arch Psychiatry Clin Neurosci 1998;248(2):96-103.

13. Dunkley EJC, Isbister GK, Sibbritt D, Dawson AH, et al. The Hunter Serotonin Toxcicity Criteria: simple and accurate diagnostic decision rules for serotonin toxicity. QJM 2003;96(9):635-42.

14. Tse L, Barr A, Scarapicchia V, et al. Neuroleptic Malignant Syndrome: A Review from a Clinically Oriented Perspective. Curr Neuropharmacol 2015;13(3):395-406.

15. Volpi-Abadie J, Kaye AM, Kaye AD. Serotonin Syndrome. Ochsner J. 2013;13(4):533-540. 